\title{
Determinación de la Respuesta Dinámica y Estacionaria de la Frecuencia frente a Desbalances de Potencia Producidos por Perturbaciones en la Red
}

\author{
N. Granda D. Colomé E. Agüero \\ Universidad Nacional de San Juan-CONICET \\ E-mail:nelson.granda@celec.gob.ec; colome@iee.unsj.edu.ar; eaguero@iee.unsj.edu.ar
}

\section{Resumen}

En este trabajo se propone una nueva metodología para determinar la respuesta dinámica y estacionaria de la frecuencia, ante desbalances de generación-carga producidos por perturbaciones en el Sistema Eléctrico de Potencia (SEP). Partiendo del modelo equivalente del sistema de control potenciafrecuencia, se derivan expresiones analíticas para calcular la máxima desviación transitoria de la frecuencia, el instante en que se produce, y la cantidad de carga a desconectar por actuación del Esquema de Alivio de Carga (EAC). Con este fin, se representa al conjunto regulador de velocidad-turbina de cada generador mediante un modelo reducido de 1er orden. En el presente trabajo, el modelo ha sido extendido para representar reguladores de unidades hidráulicas de diferente tecnología. Adicionalmente, la metodología permite determinar el valor estacionario de la frecuencia, luego de la actuación de la Regulación Primaria de Frecuencia (RPF), considerando la carga desconectada por actuación del EAC y/o el déficit de reserva rotante en unidades en RPF. Finalmente, la metodología propuesta es aplicada en dos sistemas de prueba, mostrando una excelente capacidad para estimar, con alta precisión, la respuesta dinámica y estacionaria de la frecuencia ante desbalances de potencia activa.

Palabras clave - Modelos de respuesta de la frecuencia, respuesta transitoria, frecuencia post-disturbio, regulación primaria de frecuencia, esquema alivio de carga.

\section{Abstract}

This paper proposes a new methodology for determining the transient and steady state frequency deviations produced by disturbances in the power system that modify the generationload balance. Based on the equivalent frequency response model, analytical expressions are derived to calculate the maximum transient frequency deviation, the instant it occurs, and the amount of load to be shed due to Under Frequency Load Shedding Scheme (UFLS) action. To this end, a first order model is employed to represent the governor-turbine block of each generator. In this paper, the model has been extended to represent hydro turbine speed governors of different technology. Additionally, the methodology determines the post-disturbance steady state frequency, after primary frequency control (PFC), taking into consideration the load disconnected by UFLS and spinning reserve limits of generators under PFC. Lastly, the proposed methodology is applied to two test systems, showing an excellent ability to accurately estimate the transient and steady state frequency response, due to active power imbalances.

Index terms - Frequency response model, transient response, post-disturbance frequency, primary frequency control, under frequency load shedding. 


\section{INTRODUCCIÓN}

La frecuencia es una variable fundamental en SEP y su desempeño suele estar regido mediante estándares técnicos y regulaciones operativas, debido a que las desviaciones de frecuencia fuera de la banda de operación normal afectan la confiabilidad del sistema, y posiblemente, la operación eficiente del mercado eléctrico. Los principales efectos perjudiciales de las grandes variaciones de frecuencia son: i) Daños en los equipos del sistema, especialmente generadores térmicos y transformadores, ii) Degradación de la calidad del producto entregado al usuario final (flicker), y en determinadas circunstancias, iii) El colapso parcial o total del sistema eléctrico, por actuación en cascada de protecciones de sub/ sobre frecuencia, y iv) La sobrecarga de vínculos de transmisión, debido a la respuesta de los generadores para compensar dichas variaciones [1]. La frecuencia ha sido empleada como un indicador confiable para determinar si el SEP se encuentra en un estado de emergencia, al contrario de lo que sucede con la tensión y la corriente, que son indicadores de naturaleza local.

Debido a que la máxima desviación transitoria de la frecuencia, su velocidad inicial de declinación y el nuevo valor de estado estable luego de un disturbio, son indicadores de la magnitud del desbalance generación-carga [2], es necesario contar con una metodología que permita determinar dichos valores, y al mismo tiempo, pueda ser empleada en el diseño de EAC convencionales y adaptables, el diseño de esquemas de separación controlada en islas, la parametrización de protecciones por sub/ sobre frecuencia de generadores, la determinación de reservas de generación, etc.

Varias propuestas en este sentido pueden encontrarse en la bibliografía técnica, en las que se destaca el uso de: i) Expresiones analíticas y nomogramas [3], [4], [5] de fácil uso, cálculo rápido y respuesta aproximada, ii) Simulación dinámica del modelo completo del SEP [6], [7], [8], que entrega la respuesta exacta de la frecuencia a un costo computacional que puede llegar a ser alto dependiendo del tamaño y complejidad del sistema en análisis, y iii) Modelos equivalentes del sistema de control potencia-frecuencia [9], [10], [11] cuya exactitud es muy cercana con la obtenida de una simulación dinámica a un costo computacional razonable.

Los modelos equivalentes del sistema de control potencia-frecuencia se aplican para determinar la respuesta de la frecuencia en SEP aislados; su aplicación a sistemas interconectados implica asumir que, en cada sistema, los generadores se comportan de manera coherente y por tanto la frecuencia es la misma en cada uno, pudiendo ser representada mediante la frecuencia media [12]. Una de las principales limitaciones de dichos modelos es que han sido aplicados para representar solamente a generadores térmicos y no consideran el efecto de los limitadores en las variables de estado del conjunto regulador de velocidad-turbina.

En el presente trabajo, se determina un modelo reducido de 1er orden del conjunto regulador de velocidad-turbina de cada generador, el modelo ha sido extendido para representar tanto a generadores térmicos como generadores hidráulicos con reguladores de diferente tecnología. Los parámetros del modelo reducido son obtenidos mediante el ajuste de la respuesta del modelo reducido de 1er orden a la respuesta del modelo dinámico completo del generador. A partir de los modelos reducidos de 1er orden, se determina el modelo equivalente del sistema, y, se formulan expresiones analíticas que permiten calcular: la máxima desviación transitoria de frecuencia y el instante en que se produce, el instante y cantidad de carga que se desconectará debido a la actuación del EAC. Adicionalmente, se presentan las expresiones analíticas que permiten determinar la respuesta en estacionario de la frecuencia luego de la actuación de la RPF, considerando la desconexión de carga por EAC y/o el déficit de reserva rotante en unidades en RPF.

El trabajo se organiza de la siguiente manera: en la sección 2, se presentan las ecuaciones que representan al modelo equivalente del sistema y la metodología para obtener los parámetros del modelo reducido de 1er orden de cada generador. En la sección 3, se propone una metodología de cálculo para determinar la Característica de Frecuencia del sistema y el valor de la frecuencia en estado estacionario luego de la RPF. Los resultados de la metodología propuesta son presentados en la sección 4; y finalmente, en la sección 5 se muestran las conclusiones del trabajo y recomendaciones para futuras investigaciones. 
2. MODELO DINÁMICO DEL SISTEMA DE CONTROL POTENCIAFRECUENCIA $(P-f)$

El modelo del sistema de control $P-f$ permite calcular la respuesta transitoria de la frecuencia de un SEP durante la RPF, y considera las principales características de las unidades de generación y la demanda: i) Respuesta inercial de los generadores, ii) Respuesta a la frecuencia de la demanda agregada, iii) Respuesta de los controladores $P-f$ de cada generador, es decir el conjunto regulador de velocidad-turbina, iv) EAC, y (v) Desbalance de generación-carga. El diagrama de bloques del modelo del sistema control $P-f$ se muestra en la Fig. 1.

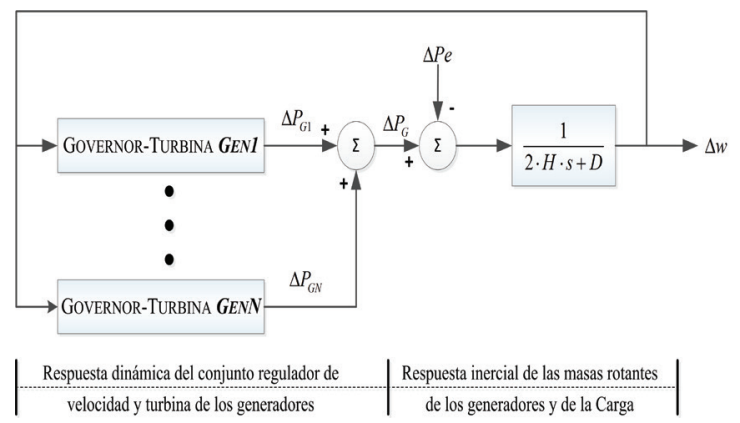

Figura 1: Modelo del Sistema de control $P$ - $f$

\subsection{Modelo equivalente del sistema de control $P-f$}

La respuesta dinámica de la frecuencia, luego de un desbalance de generación-carga $\triangle P o(s)$, puede ser calculada mediante un modelo equivalente que considera las características de regulación de frecuencia del sistema: la inercia equivalente del sistema $\left(H_{e q}\right)$, el estatismo $\left(R_{i}\right)$ de cada generador, y el amortiguamiento de la carga $(D)$. El modelo considera también el conjunto regulador de velocidad-turbina de cada unidad de generación, a través de un modelo reducido de 1er orden [11]. La Fig. 2 muestra el diagrama de bloques del modelo equivalente de un SEP con $\mathrm{N}$ generadores.

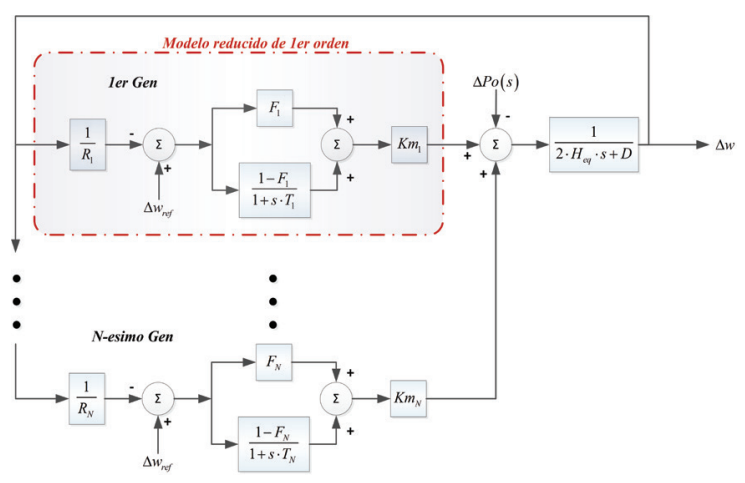

Figura 2: Modelo equivalente del sistema de control $P$ - $f$
Empleando el modelo equivalente descrito, se obtiene la respuesta dinámica de la frecuencia en el dominio de Laplace, ante una variación escalón en la carga $\triangle P o(s)$, mediante .

$$
\begin{aligned}
& \Delta w(s)=-\Delta P o(s) \cdot \frac{g_{1}(s)}{g_{2}(s)} \\
& g_{1}(s)=\prod_{i=1}^{N}\left(1+s T_{i}\right) \\
& g_{2}(s)=\left(2 s H_{e q}+D\right) \cdot \prod_{i=1}^{N}\left(1+s T_{i}\right)+\sum_{j=1}^{N}\left[\frac{K m_{j}}{R_{j}}\left(1+F_{j} T_{j} s\right) \cdot \prod_{\substack{i=1 \\
i \neq j}}^{N}\left(1+s T_{i}\right)\right]
\end{aligned}
$$

Dónde: $K m_{i}, F_{i}$ y $T_{i}$ son los parámetros del modelo reducido de ler orden del conjunto regulador de velocidad-turbina de cada generador [10].

La respuesta temporal del modelo equivalente del sistema de control $P-f$, se obtiene realizando la expansión en fracciones parciales de y aplicando la anti transformada de Laplace. Los resultados se pueden resumir mediante.

$$
\Delta w(t)=\Delta P o \cdot \sum_{i=1}^{N+1} \frac{A_{i}}{p_{i}}\left(1-e^{p_{i} t}\right) \cdot U(t)
$$

Dónde: $A_{i}$ y $p_{i}$ son los residuos y polos respectivamente, resultantes de la expansión en fracciones parciales. El instante de tiempo $t\left(\Delta f_{\max }\right)$ en que ocurre la máxima desviación de la frecuencia se obtiene derivando e igualando a cero la expresión. Una vez obtenido $t\left(\Delta f_{\max }\right)$, se lo reemplaza en para obtener la máxima desviación transitoria de la frecuencia $\left(\Delta f_{\max }\right)$.

En el modelo equivalente mencionado, es posible incluir un Esquema de Alivio de Carga (EAC) y analizar sus efectos en el desempeño dinámico de la frecuencia. La activación de cada escalón del EAC modifica el desbalance de potencia activa $\triangle P o$ en la expresión, y por lo tanto, la máxima desviación transitoria de la frecuencia.

\subsection{Modelo reducido de $1 \mathrm{er}$ orden de los generadores}

El modelo reducido de 1er orden es una simplificación de los modelos usualmente complejos que se emplean para obtener la respuesta dinámica del conjunto regulador de velocidadturbina de cada generador, y sus parámetros son determinados mediante el ajuste de la repuesta del modelo reducido a la respuesta del modelo dinámico completo ante variaciones en la carga 
local que alimenta el generador, como puede observase en la Fig. 3.

El ajuste de ambas respuesta se realiza aplicando un algoritmo de optimización, en este trabajo el método de mínimos cuadrados no lineales incluido en la librería de identificación de parámetros [13] de MATLAB $\AA$, cuya función objetivo es minimizar la diferencia entre ambas respuestas.

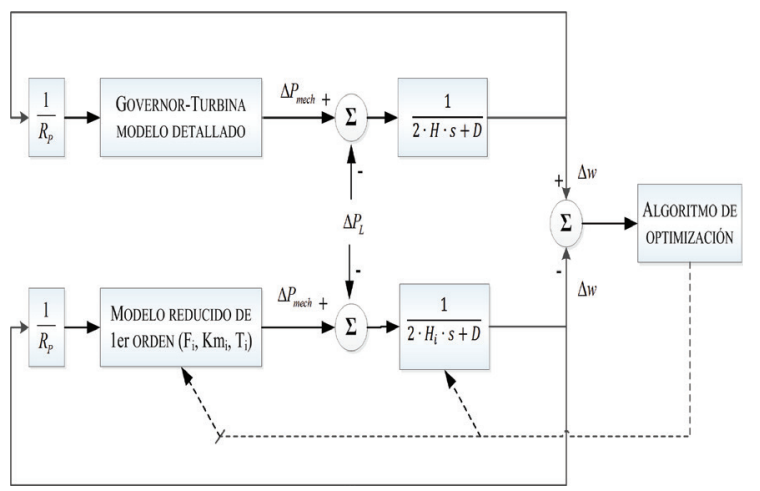

Figura 3: Determinación parámetros modelo reducido 1er orden

En [11] se obtienen los parámetros $K m_{i}, F_{i}$ y $T_{i}$ correspondientes a unidades térmicas. En este trabajo se extiende el modelo a unidades hidráulicas, donde para lograr el ajuste requerido, es necesario considerar al momento de inercia del generador como nueva variable de ajuste.

El ajuste entre las respuestas del modelo reducido de 1 er orden y del modelo completo se realiza hasta el instante de tiempo en que usualmente ocurre la máxima desviación transitoria de la frecuencia, que se puede conocer de experiencia previa acerca del comportamiento dinámico del SEP.

\section{MODELO ESTACIONARIO DEL CONTROLPOTENCIA-FRECUENCIA $(P-f)$}

Para calcular el valor de la frecuencia en estado estacionario luego de la actuación de la RPF, producto de un desbalance de potencia activa, se propone un procedimiento de cálculo que incluye las restricciones de reserva rotante de los generadores bajo RPF. El procedimiento contempla además el efecto del EAC en el desbalance de potencia activa.

\subsection{Característica de frecuencia}

La curva que define la capacidad de regulación de frecuencia en estado estacionario de un SEP, $\mathrm{y}$ por tanto el estatismo permanente equivalente
$\left(R_{E Q}\right)$ del mismo, se denomina Característica de Frecuencia [14], y es dependiente del estatismo permanente de los reguladores de las unidades que efectivamente aportan a la RPF. La potencia que puede entregar la turbina de un generador está limitada por sus parámetros técnicos y cuando la unidad de generación ha alcanzado sus límites de generación $\left(P G_{M A X}\right.$ y $P G_{M I N}$ ), es incapaz de seguir aportando a la RPF. Es así que en general, la Característica de Frecuencia es una curva no lineal, cuya pendiente crece a medida que las unidades en RPF alcanzan sus límites de potencia.

El cálculo de la Característica de Frecuencia se realiza de la siguiente manera:

i) Se determina el rango de regulación de cada generador $G_{i}$, mediante, donde $P G_{i}$ es la potencia generada a frecuencia nominal $\left(f_{\text {nom }}\right)$ y $R_{\text {real-Gi }}$ representa el estatismo del generador $i$ expresado en (Hz/MW).

$$
\begin{aligned}
& f_{\text {min-Gi }}=f_{\text {nom }}-R_{\text {real }-G i}\left(P G_{M A X-G i}-P G_{i}\right) \\
& f_{\text {max }-G i}=f_{\text {nom }}+R_{\text {real }-G i}\left(P G_{i}-P G_{M I N-G i}\right)
\end{aligned}
$$

ii) Se construye el vector $\mathbf{F}_{\text {LIM }}$, que está constituido por los límites de frecuencia en que regulan los generadores, los límites máximos y mínimos de actuación de los relés de protección y la frecuencia nominal de operación.

$$
\mathbf{F}_{L I M}=\left[\begin{array}{c}
f_{\text {rel-MIN }} \\
f_{\text {min-G }} \\
\mathrm{M} \\
f_{\text {nom }} \\
f_{\min -G_{N}} \\
f_{\max -G_{1}} \\
\mathrm{M} \\
f_{\max -G_{N}} \\
f_{r e l-M A X}
\end{array}\right]
$$

iii) Se construye la matriz $\mathbf{R}_{\text {MAT }}$, de $N_{G E N}$ columnas (cantidad de generadores) y $N_{L I M}$ filas (dimensión de $\mathbf{F}_{\text {LIM }}$ ). Las columnas se llenan con unos (1) en el rango de regulación de cada generador y ceros $(0)$ en caso contrario. La matriz $\mathbf{R}_{\text {MAT }}$ permite identificar el rango de frecuencia en que cada generador y el sistema en general es capaz de realizar la regulación de frecuencia. 


$$
\begin{aligned}
& \begin{array}{lllll}
G_{1} & G_{2} & \mathrm{~L} & G_{N}
\end{array} \\
& \mathbf{R}_{M A T}=\left[\begin{array}{cccc}
0 & 1 & \mathrm{~L} & 0 \\
1 & 1 & \mathrm{~L} & 0 \\
1 & 1 & \mathrm{~L} & 0 \\
1 & 1 & \mathrm{~L} & 1 \\
1 & 1 & \mathrm{~L} & 1 \\
1 & 0 & \mathrm{~L} & 1 \\
0 & 0 & \mathrm{~L} & 1 \\
0 & 0 & \mathrm{~L} & 1
\end{array}\right] \begin{array}{c}
f_{\min -G_{2}} \\
f_{\min -G_{1}} \\
f_{\min -G_{N}} \\
f_{\max -G_{2}} \\
f_{\max -G_{1}} \\
f_{\max -G_{N}}
\end{array}
\end{aligned}
$$

iv) Multiplicando la matriz $\mathbf{R}_{\text {MAT }}$ por el vector de estatismos permanentes individuales $\mathrm{R}_{G i}$ se obtiene el vector de $\mathbf{R}_{\mathrm{EQ}}$, el cual contiene el estatismo equivalente del SEP en el rango de regulación de las unidades.

$$
\frac{1}{\mathbf{R}_{E Q}}=\mathbf{R}_{M A T} *\left[\begin{array}{llll}
\frac{1}{R_{G_{1}}} & \frac{1}{R_{G_{2}}} & \mathrm{~L} & \frac{1}{R_{G_{N}}}
\end{array}\right]^{T}
$$

v) La potencia de reserva efectiva disponible a lo largo de la Característica de Frecuencia del SEP se obtiene al calcular el vector $\mathbf{P}_{\text {EQ }}$, de $N_{L I M}$ filas, mediante .

$$
\begin{aligned}
\mathbf{P}_{E Q}= & \mathbf{P}_{1} * \mathbf{P G}_{M A X}+\operatorname{diag}\left(\mathbf{P}_{2} * \mathbf{P G}(f)\right) \\
& +\mathbf{P}_{3} * \mathbf{P}_{M I N}
\end{aligned}
$$

Dónde, las matrices $\mathbf{P}_{1}, \mathbf{P}_{2}, \mathbf{P}_{3}$ están formadas por unos (1) y ceros (0), con una estructura similar a la matriz $\mathbf{R}_{\mathrm{MAT}}$. Cuando, a lo largo del rango de regulación de frecuencia del sistema, un generador se encuentre en su potencia máxima $\left(P G_{M A X}\right)$, dentro de sus límites de operación normal $(P G(f))$ o en su potencia mínima $\left(P G_{M I N}\right)$, se ingresará el valor uno (1) en las matrices $\mathbf{P}_{1}, \mathbf{P}_{2} \circ \mathbf{P}_{3}$, respectivamente.

vi) Finalmente, se determina la Característica de Frecuencia (matriz $\mathbf{F C}_{\mathbf{M A T}}$ ), mediante .

$$
\mathbf{F C}_{M A T}=\left[\begin{array}{lll}
\mathbf{F}_{L I M} & \mathbf{R}_{E Q} & \mathbf{P}_{E Q}
\end{array}\right]
$$

\subsection{Determinación de la frecuencia en estado estacionario}

La variación de la frecuencia en estado estacionario $\Delta f_{s s}$, luego de la actuación de la RPF, se calcula mediante ; donde, $R_{E Q}$ es el estatismo permanente equivalente, $D$ es el coeficiente de variación de la carga con la frecuencia y $\Delta P$ el desbalance de potencia activa.

$$
\begin{aligned}
& f_{s s}=f_{\text {nom }}+\Delta f_{s s} \\
& \Delta f_{s s}=-\frac{R_{E Q}}{1+R_{E Q} \cdot D} \cdot \Delta P
\end{aligned}
$$

El procedimiento propuesto contempla las restricciones de reserva rotante de las unidades que participan en RPF. Cuando un generador $G_{i}$ alcanza su valor máximo de generación durante la RPF, antes de que la frecuencia alcance su valor de estado estacionario, el desbalance efectivo de potencia se calcula mediante . Por otra parte, el cálculo de la máxima desviación transitoria de la frecuencia, descrito en la sección 2, permite identificar los pasos del EAC que se han activado y corregir el desbalance de potencia activa, contemplando el porcentaje de carga desconectada $\triangle P_{E A C}$, como indica .

$$
\Delta P=\Delta P_{0}-\left(P G_{M A X-G i}-P G_{i}\right)-\Delta P_{E A C}
$$

\section{RESULTADOS}

\subsection{Modelo reducido - Unidades hidráulicas}

Utilizando la librería de identificación de parámetros de MATLAB ${ }^{\circledR}$ se obtienen los modelos reducidos de 1er orden para los modelos de reguladores de velocidad de turbinas hidráulicas IEEEG2, IEEEG3 y HYGOV [15], [16]. En la Tabla 1 se presentan los valores de las constantes $K m_{i}, F_{i}, T_{i}, H_{i}$ y del coeficiente de determinación $R^{2}$, que mide el ajuste entre los modelos completo y reducido de 1er orden. Para el regulador de velocidad HYGOV debido a la alta influencia del modelo no lineal de la turbina hidráulica se han incluido dos ajustes considerando dos estados de operación representativos. 
Tabla 1: Contantes de los modelos reducidos de unidades hidráulicas

\begin{tabular}{|c|c|c|c|c|c|}
\hline \multicolumn{1}{c}{ Función de } & \multicolumn{7}{c|}{ Constantes del Modelo } \\
Transferencia & $\boldsymbol{T}_{\boldsymbol{i}}$ & $\boldsymbol{F}_{\boldsymbol{i}}$ & \multicolumn{1}{c|}{$\boldsymbol{K}_{\boldsymbol{i}}$} & $\boldsymbol{H}_{\boldsymbol{i}}$ & $\boldsymbol{R}^{\mathbf{2}}$ \\
\hline HYGOV $\left(\mathrm{Pm}_{0}=0.9\right)$ & 3,108 & $-0,651$ & 0,103 & 3,146 & 0,999 \\
\hline HYGOV $\left(\mathrm{Pm}_{0}=0.5\right)$ & 2,984 & $-0,303$ & 0,109 & 2,955 & 0,999 \\
\hline IEEEG2 & 0,913 & $-1,354$ & 0,166 & 3,221 & 0,999 \\
\hline IEEEG3 & 4,547 & $-0,351$ & 0,169 & 3,274 & 0,998 \\
\hline
\end{tabular}

En la Fig. 4 se muestran la respuesta de la frecuencia de los modelos completo y reducido de 1er orden para los modelos antes mencionados, frente a un aumento de carga. El tiempo de simulación para realizar el ajuste depende de las constantes de tiempo que intervienen en cada modelo. Las simulaciones se realizan en SIMULINK $®$. Se observa en los tres casos que la respuesta del modelo reducido presenta un muy buen ajuste a la respuesta del modelo completo en el periodo de tiempo donde se alcanza el mínimo valor de la frecuencia.

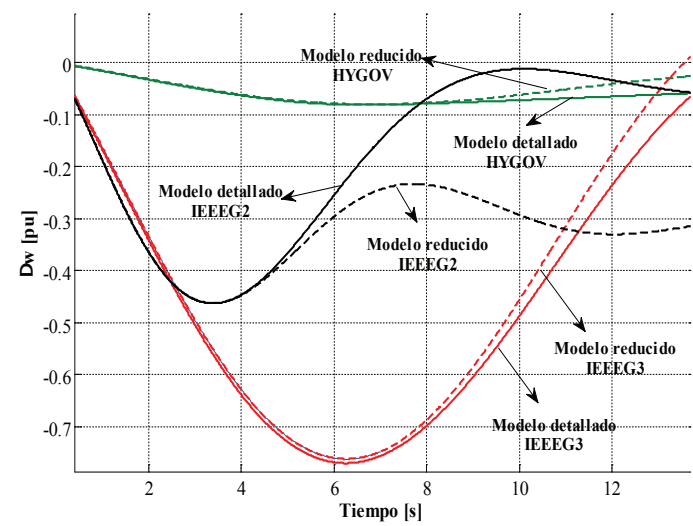

Figura 4: Respuesta de frecuencia de los modelos completo y reducido - Unidades hidráulicas

\subsection{Respuesta del modelo equivalente}

En el sistema WSCC de 9 barras -3 generadores [17] se analiza y compara la respuesta de los siguientes modelos:

- Modelo con toda la dinámica. Simulación mediante PowerWorld Simulator (PWS).

- Modelo completo del sistema de control P-f. Simulación mediante SIMULINK ${ }^{\circledR}$.

- Modelo equivalente del sistema de control $P-f$. Simulación mediante SIMULINK®.

- Modelo analítico del sistema de control $P$ - $f$. Valores de $\Delta f_{M A X}$ y $t\left(\Delta f_{M A X}\right)$ obtenidos mediante MATLAB®.

En los tres últimos casos se modela una única ecuación de movimiento que representa la respuesta de los tres generadores y de la carga ante desbalances de potencia activa, con una constante de inercia equivalente calculada mediante .

$H_{E Q}=H_{1} \cdot \frac{\text { Snom }_{1}}{S_{\text {base }}}+H_{2} \cdot \frac{\text { Snom }_{2}}{S_{\text {base }}}+H_{3} \cdot \frac{\text { Snom }_{3}}{S_{\text {base }}}$

En la Fig. 5, se muestra la respuesta obtenida mediante cada modelo. La frecuencia en cada barra, obtenida mediante simulación transitoria empleando PWS, se muestra con líneas grises. Con línea verde se representa la evolución de la frecuencia obtenida mediante el modelo completo del sistema de control $P-f$, y con línea azul se representa la evolución de la frecuencia obtenida mediante el modelo equivalente. La desviación transitoria máxima de la frecuencia, obtenida mediante el modelo analítico, se representa mediante un rombo azul $(\diamond)$.

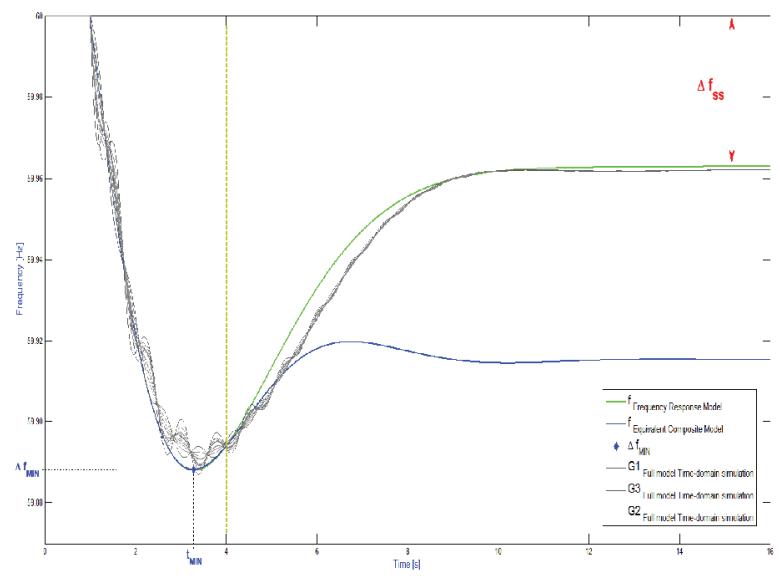

Figura 5: Respuesta de la frecuencia. PWS, modelos completo y equivalente

Para obtener el modelo reducido de cada generador se considera un periodo de ajuste de $4,0 \mathrm{~s}$. Se puede observar que hasta los $4,0 \mathrm{~s}$, la evolución temporal del modelo completo y del modelo equivalente del sistema de control $P-f$ es idéntica; sin embargo, después de este momento ambas respuestas empiezan a alejarse entre sí. La respuesta transitoria de ambos modelos se ajusta bien con la evolución de la frecuencia obtenida mediante simulación transitoria en PWS. Finalmente, la desviación de frecuencia de estado estacionario $\left(\Delta f_{s s}\right)$, obtenida mediante, es muy similar a la frecuencia de estado estacionario obtenida mediante PWS.

\subsection{Desconexión de carga por actuación del EAC}

Sobre el mismo sistema de prueba, se simula la pérdida de $114,1 \mathrm{MW}$ en el generador $G_{2}$, 
ocasionando la actuación del EAC hasta el tercer umbral. En la Fig. 6 se presenta la evolución de la frecuencia obtenida mediante: i) El modelo equivalente del sistema, ii) Las expresiones analíticas derivadas en la sección 2, considerando la actuación del EAC, y iii) Simulación transitoria en PWS. Puede observarse que el comportamiento de la frecuencia, obtenido mediante los modelos equivalente y analítico, es muy similar al obtenido mediante PWS, cuando se considera la frecuencia mínima y su instante de ocurrencia.

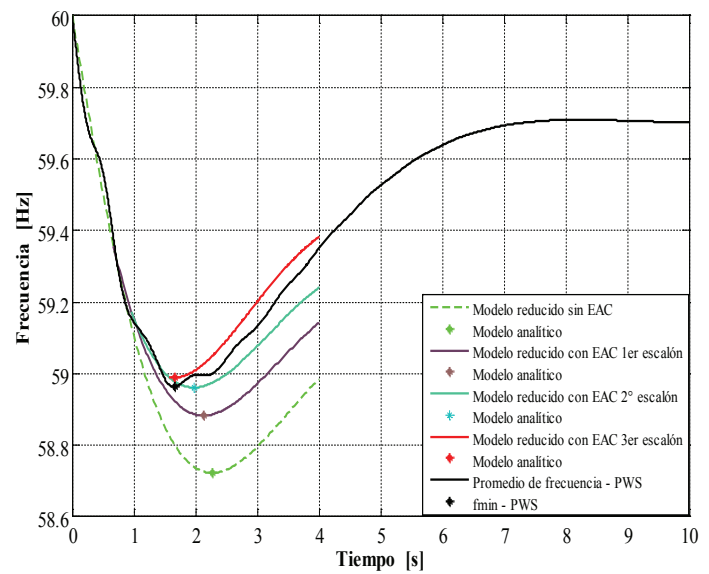

Figura 6: Evolución de la frecuencia contemplando la actuación del EAC

En la Tabla 2 se muestran los valores de frecuencia mínima y tiempo de ocurrencia obtenidos mediante el modelo analítico y PWS; se presenta también los errores considerando como referencia la frecuencia media del sistema obtenida mediante PWS. Se puede observar que los valores de frecuencia mínima son similares, aproximadamente $-0,04 \%$ de error, mientras que el error en el tiempo es de $-0,3 \%$.

Tabla 2: Comparación entre modelos

\begin{tabular}{|c|c|c|c|c|} 
Modelo & $\boldsymbol{f}_{\min }[\mathrm{Hz}]$ & \multicolumn{1}{c}{$\left(\boldsymbol{f}_{\text {min }}\right)[s]$} & $\begin{array}{c}\boldsymbol{E R}\left(\boldsymbol{f}_{\text {min }}\right) \\
\boldsymbol{\%}\end{array}$ & $\begin{array}{c}\boldsymbol{E R}\left(\boldsymbol{t}\left(\boldsymbol{f}_{\text {min }}\right)\right. \\
\boldsymbol{\%}\end{array}$ \\
\hline PWS & 58,96402 & 1,6583 & - & - \\
\hline Analítico & 58,98969 & 1,6634 & $-0,0435$ & $-0,3079$ \\
\hline
\end{tabular}

\subsection{Cálculo de la característica de frecuencia}

La Fig. 7 muestra la Característica de Frecuencia del sistema WSCC, donde se considera que los generadores $G_{1}$ y $G_{2}$ son unidades térmicas (TGOV1) y el generador $G_{3}$ es una unidad hidráulica (IEEG2). El conjunto de generadores es capaz de regular la frecuencia entre los 58,0 y $61,1 \mathrm{~Hz}$. Se puede observar que alrededor de la frecuencia nominal, las 3 unidades participan en la RPF. Los cambios de pendiente indican que las unidades van agotando su reserva rotante y que el estatismo equivalente del sistema ha cambiado.

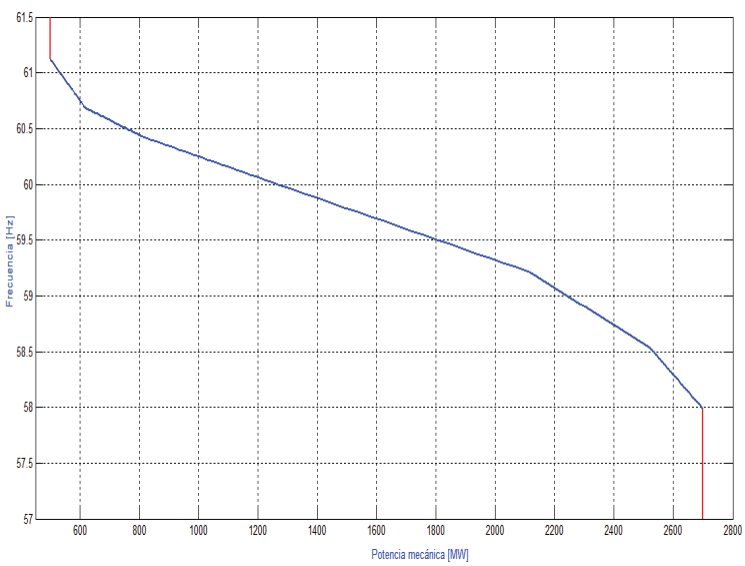

Figura 7: Característica de frecuencia - sistema WSCC

\subsection{Cálculo de la frecuencia de estado estacionario}

Se calcula la frecuencia en estado estacionario ante aumentos de carga de: a) $20 \mathrm{MW}$ con las 3 unidades en RPF, sin restricciones de reserva $\left(R_{E Q}\right.$ $=1 / 170)$, b) $50 \mathrm{MW}$ que provoca que la unidad $G_{3}$ agote su reserva $\left(R_{E Q}=1 / 150\right)$, y c) $70 \mathrm{MW}$ que además de agotar la reserva de $G_{3}$ produce la actuación del 1er escalón de EAC. Los resultados obtenidos con el modelo analítico y mediante SIMULINK ${ }^{\circledR}$ se presentan en la Fig. 8.

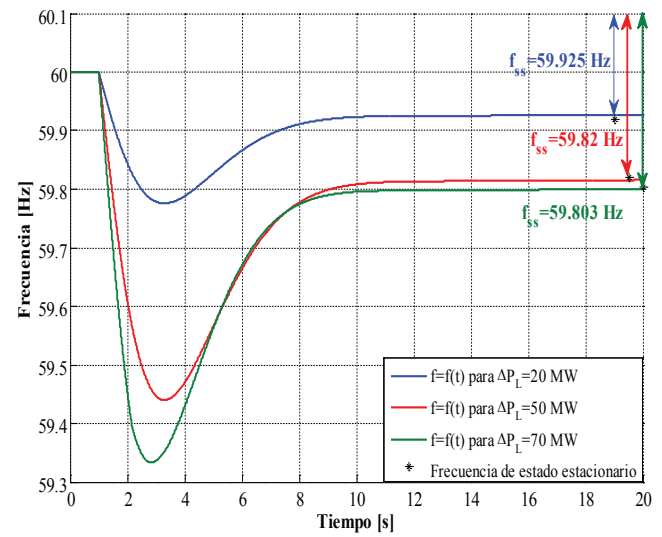

Figura 8: Frecuencia en estado estacionario ante diferentes perturbaciones

\subsection{Aplicación de modelo $P$ - $f$ en desconexión adaptable de carga}

En [18], los autores proponen un nuevo Esquema de Separación Controlada en Islas eléctricas (ESCI) que incluye una etapa de desconexión adaptable de carga, diseñada para controlar la frecuencia en cada isla. Para determinar la cantidad de carga a 
desconectar, se emplea el modelo equivalente del sistema de control $P$ - $f$. La cantidad de carga a desconectar asegura que el desbalance de potencia activa en cada isla no provoque variaciones transitorias de frecuencia que induzcan la actuación del EAC.

Ante una serie de eventos en cascada y con el objetivo de evitar el colapso parcial del sistema, la aplicación del ESCI propuesto conduce a la separación del sistema New England de 39 barras - 10 generadores [19] en dos islas eléctricas, la primera con déficit de generación, mientras que la segunda con superávit de potencia activa. En la Fig. 9 se muestra la evolución dinámica de la frecuencia obtenida mediante el modelo equivalente del sistema de control $P-f$. Se observa que en la Isla 1, la frecuencia mínima es menor a los 59,4 Hz (línea llena celeste), lo que provoca la actuación del primer escalón del EAC. Mediante el modelo equivalente se determina la cantidad de carga a desconectar, para lograr que el nuevo desbalance en la isla no provoque la actuación del EAC convencional. En la Isla 2, la máxima desviación transitoria de la frecuencia no activa las protecciones de sobre frecuencia (línea verde). La máxima desviación transitoria de la frecuencia en cada isla, obtenida mediante el modelo analítico, se representa mediante rombos $(\diamond)$.

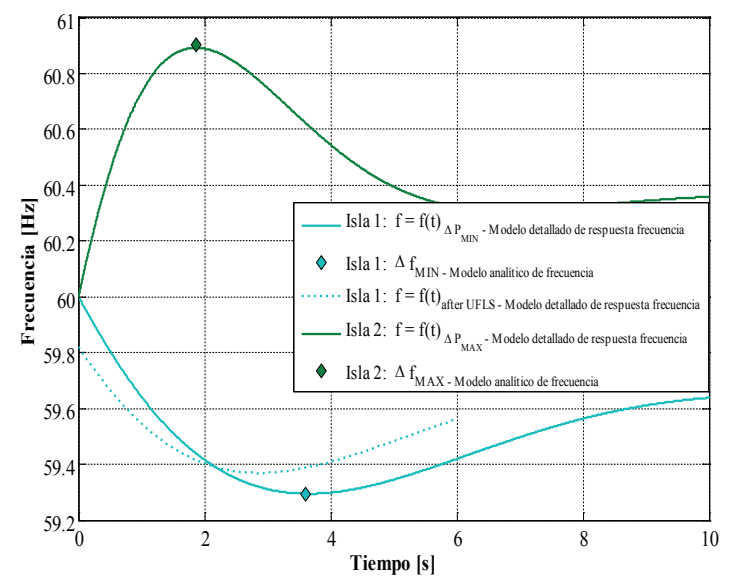

Figura 9: Desviación de frecuencia en Isla 1 e Isla 2

\section{CONCLUSIONES RECOMENDACIONES}

El comportamiento de la frecuencia del sistema, luego que ha ocurrido un disturbio, puede ser exitosamente determinada mediante el modelo equivalente del sistema de control $P$-f. Esto se verifica comparando la respuesta del sistema cuando se emplean los modelos completos del conjunto regulador de velocidad - turbina, respecto de la obtenida cuando se emplea el modelo equivalente conformado por los modelos reducidos de 1 er orden.

La determinación de la máxima desviación transitoria de la frecuencia, su instante de ocurrencia y la desviación de frecuencia en estado estacionario permiten desarrollar esquemas de emergencia para controlar la frecuencia en el sistema, luego de un disturbio que modifique el balance generación-carga.

La determinación del modelo reducido de 1er orden de los generadores del sistema, puede llegar a ser un trabajo largo y computacionalmente costoso; sin embargo, se lo realiza fuera de línea y una sola vez por generador. La determinación de la Característica de Frecuencia del sistema es una información valiosa para la operación en tiempo real, pues permite al operador conocer el estatismo equivalente real $\left(R_{E Q}\right)$ del sistema, y puede, inclusive, ser aplicada para actualizar el factor Bias ( $\beta$ ) del AGC.

Para asegurar que la respuesta del modelo equivalente represente de manera fidedigna la evolución de la frecuencia del sistema, es necesario validar a través de ensayos en campo los modelos completos de los generadores, a partir de los cuales se obtienen los modelos reducidos de 1er orden.

En trabajos futuros, se debe investigar $y$ desarrollar una metodología que permita incluir el efecto de los limitadores en el modelo analítico presentado.

\section{REFERENCIAS BIBLIOGRÁFICAS}

[1] B. J. Kirby, J. Dyer, C. Martinez, R. A. Shoureshi, R. Guttromson, and J. Dagle, "Frequency control concerns in the North American electric power system," U.S. Department of Energy, Oak Ridge, US, Technical Report ORNL/TM-2003/41,2002.

[2] V. V. Terzija, "Adaptive underfrequency load shedding based on the magnitude of the disturbance estimation," Power Systems, IEEE Transactions on, vol. 21, pp. 12601266, 2006.

[3] L. L. Fountain and J. L. Blackburn, "Application and Test of Frequency Relays for Load Shedding," Power Apparatus and Systems, Part III. Transactions of the American Institute of Electrical Engineers, vol. 73, pp. 1660-1668, 1954. 
[4] C. F. Dalziel and E. W. Steinback, "Underfrequency Protection of Power Systems for System Relief Load SheddingSystem Splitting," Power Apparatus and Systems, Part III. Transactions of the American Institute of Electrical Engineers, vol. 78, pp. 1227-1237, 1959.

[5] H. E. Lokay and V. Burtnyk, "Application of Underfrequency Relays for Automatic Load Shedding," Power Apparatus and Systems, IEEE Transactions on, vol. PAS-87, pp. 776$783,1968$.

[6] C. Evrard and A. Bihain, "Powerful tools for various types of dynamic studies of power systems," in Proceedings of 1998 International Conference on Power System Technology, POWERCON 1998, pp. 1-6 vol.1.

[7] DIgSILENT GmbH, "DIgSILENT PowerFactory User Manual,” ed. Gomaringen, Germany,: DIgSILENT GmbH, 2013.

[8] Siemens Power Transmission \& Distribution Inc, "PSS/E 30.2 Users Manual," ed. Schenectady, US: Siemens Power Transmission \& Distribution Inc, 2005.

[9] P. M. Anderson and M. Mirheydar, "A loworder system frequency response model," Power Systems, IEEE Transactions on, vol. 5, pp. 720-729, 1990.

[10]A. Denis Lee Hau, “A general-order system frequency response model incorporating load shedding: analytic modeling and applications," Power Systems, IEEE Transactions on, vol. 21, pp. 709-717, 2006.

[11]I. Egido, F. Fernandez-Bernal, P. Centeno, and L. Rouco, "Maximum Frequency Deviation Calculation in Small Isolated Power Systems," Power Systems, IEEE Transactions on, vol. 24, pp. 1731-1738, 2009.

[12]H. Bevrani, Robust Power System Frequency Control, 2nd ed. Switzerland: Springer, 2014.

[13]L. Ljung. (2015). System Identification Toolbox: User's Guide (Online version ed.). Available: http://www.mathworks.com/help/ pdf_doc/ident/ident.pdf
[14]J. Machowski, J. Bialek, and J. Bumby, Power system dynamics: stability and control, 2nd ed.: John Wiley \& Sons, 2011.

[15]Working Group on Prime Mover and Energy Supply Models for System Dynamic Performance Studies, "Hydraulic turbine and turbine control models for system dynamic studies," Power Systems, IEEE Transactions on, vol. 7, pp. 167-179, 1992.

[16]L. N. Hannett, J. W. Feltes, and B. Fardanesh, "Field tests to validate hydro turbinegovernor model structure and parameters," Power Systems, IEEE Transactions on, vol. 9, pp. 1744-1751, 1994.

[17]M. Pavella, D. Ernst, and D. Ruiz-Vega, Transient stability of power systems: a unified approach to assessment and control: Springer Science \& Business Media, 2012.

[18]N. Granda and D. G. Colome, "An Intelligent Controlled Islanding Scheme for Power Systems," in Intelligent System Applications to Power Systems (ISAP), 18th Conference on, Porto, Portugal, 2015.

[19]M. A. Pai, Energy Function Analysis for Power System Stability. Boston, USA: Kluwer Academic Publishers, 1989.

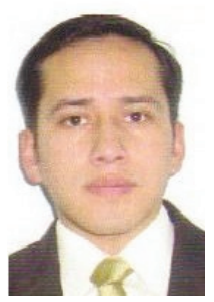

Nelson Granda Gutiérrez.Obtuvo el título de Ingeniero Eléctrico en la Escuela Politécnica Nacional de Quito, en 2006. Se ha desempeñado como Ingeniero Eléctrico en el Centro Nacional de Control de Energía (CENACE) y posteriormente en el departamento de Optimización de la Generación Eléctrica (OGE) de Petroamazonas EP.

Actualmente se encuentra realizando su trabajo de investigación previo a la obtención de título de $\mathrm{PhD}$ en el Instituto de Energía Eléctrica de la Universidad Nacional de San Juan (IEE - UNSJ). Sus áreas de interés son análisis y control de sistemas de potencia en tiempo real. Actualmente trabaja en CELEC EP, Unidad de Negocio TRANSELECTRIC. 


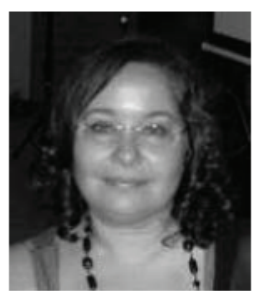

Delia Graciela Colomé.-

Obtuvo su título de Ingeniera

Electrónica en 1985 y de

Doctora en Ingeniería en 2009 Universidad Nacional de San Juan (UNSJ), Argentina. Desde 1983 trabaja en el Instituto de Energía Eléctrica (IEE) unidad de doble dependencia UNSJ-CONICET. Es coordinadora de la carrera ingeniería eléctrica y profesora en sus programas de maestría y doctorado. Es directora de proyectos de investigación y de tesis doctorales. Sus líneas de investigación comprenden la modelación, simulación supervisión, estabilidad y control de los sistemas de potencia. Ha trabajado en numerosos convenios de asistencia técnica para empresas del sector eléctrico de Argentina y de Latinoamérica.

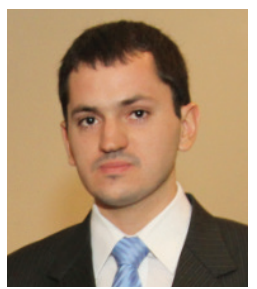

Esteban Daniel Agüero Meni.Nació en San Juan, Argentina en 1987. Obtuvo su título de Ingeniero Eléctrico en 2012 en la Universidad Nacional de San Juan, Argentina. Actualmente es un candidato a doctorado en el Instituto de Energía Eléctrica de la Universidad Nacional de San Juan, favorecido con una beca del Consejo Nacional de Investigaciones Científicas y Técnicas (CONICET). Sus campos de investigación están relacionados con estabilidad, control y protección del sistema eléctrico de potencia. 